\title{
Thickness of human articular cartilage in joints of the lower limb
}

\author{
D E T Shepherd, B B Seedhom
}

\begin{abstract}
Objectives-(a) To determine the topographical variations in cartilage thickness over the entire surfaces of cadaveric lower limb joints, and (b) to examine the correlations between: cartilage thickness and its site specific modulus; cartilage thickness and donor age, weight, height, and body mass index.
\end{abstract}

Methods-The cartilage thickness of 11 sets of cadaveric human joints each comprising an ankle, knee, and hip was measured using a needle probe technique. Statistical analysis was used to compare the cartilage thickness of the different lower limb joints and the differences in cartilage thickness over the surface of individual joints. It was further examined whether cartilage had a correlation with its stiffness, and any of the details of the specimen donors such as age, weight, height, and body mass index.

Results-The mean cartilage thickness of the knee was significantly greater than that of the ankle and hip $(p<0.001)$ in all 11 sets of joints, while the cartilage thickness of the hip was significantly greater than that of the ankle in 10 sets of joints $(p<0.001)$. The mass of specimen donors was found to correlate with the mean cartilage thickness of all three lower limb joints. A correlation was also found between the height of donors and the mean cartilage thickness of the knee and hip joints, while only in the ankle joint was a correlation found between the mean cartilage thickness and the body mass index of the specimen donors. A further correlation was found between cartilage thickness and its modulus; the thinner the cartilage, the higher the modulus.

Conclusions-The thickness of articular cartilage seems to be related to the congruance of a joint; thin cartilage is found in congruent joints such as the ankle, whereas thick cartilage is found in

Accepted for publicatio 10 September 1998

Table 1 Details of specimen donors relevant to the study

\begin{tabular}{llllll}
\hline Set number & Sex & Age $(y)$ & Mass $(\mathrm{kg})$ & Height $(\mathrm{m})$ & Body mass index $\left(\mathrm{kg} / \mathrm{m}^{2}\right)$ \\
\hline 1 & $\mathrm{~F}$ & 62 & 60 & 1.75 & 19.6 \\
2 & $\mathrm{M}$ & 68 & 50 & 1.75 & 16.3 \\
3 & $\mathrm{~F}$ & 33 & 50 & 1.70 & 17.3 \\
4 & $\mathrm{~F}$ & 65 & 74 & 1.75 & 24.2 \\
5 & $\mathrm{~F}$ & 46 & 35 & 1.60 & 13.7 \\
6 & $\mathrm{~F}$ & 80 & 64 & 1.70 & 22.1 \\
7 & $\mathrm{~F}$ & 79 & 50 & 1.52 & 21.6 \\
8 & $\mathrm{~F}$ & 70 & 48 & 1.63 & 18.1 \\
9 & $\mathrm{M}$ & 63 & 60 & 1.68 & 21.3 \\
10 & $\mathrm{M}$ & 74 & 68 & 1.75 & 22.2 \\
11 & $\mathrm{~F}$ & 76 & 40 & 1.60 & 15.6 \\
Mean & & 65.1 & 54.5 & 1.68 & 19.3 \\
SD & & 14.3 & 11.9 & 0.08 & 3.3 \\
\hline
\end{tabular}

incongruent joints such as the knee. The correlations in this study imply that the larger and heavier was a donor the thicker was the cartilage in the lower limb joints. The data further suggest the presence of an inverse relation between the mean cartilage thickness and mean compressive modulus in each of the joints examined. (Ann Rheum Dis 1999;58:27-34)

A vast array of techniques exist for measuring the thickness of articular cartilage. These methods include destructive techniques where the thickness of plugs or slices of cartilage are measured $^{1-4}$ or using a needle probe ${ }^{5}$ and nondestructive techniques such as ultrasound. ${ }^{6-8}$

Jurvelin et al did a comparison of optical, needle probe, and ultrasonic techniques for the measurement of articular cartilage thickness and concluded that each method had its limitations. One serious limitation was with ultrasound by having to assume a constant velocity of sound when it can vary greatly between cartilage specimens. Yao and Seedhom ${ }^{10}$ have shown that the velocityof sound in cartilage can vary as much as $33.6 \%$ of the average calculated from a large number of sites, leading to errors in thickness of the same percentage when compared with data obtained by direct measurement using the needle probe technique.

More recent techniques used to measure the thickness of articular cartilage have included using magnetic resonance imaging. ${ }^{112}$ Kladny et $a l^{11}$ found that the mean percentage difference between cartilage thickness in magnetic resonance imaging and direct measurement from histological sections was about $10 \%$, with the results most accurate for cartilage thicker than $2 \mathrm{~mm}$.

Given the vast array of techniques that exist for measuring the thickness of articular cartilage it is surprising that few studies have been published giving detailed measurements of the thickness of human articular cartilage in joints of the lower limb. The thickness of articular cartilage influences both the stresses and strains arising within the cartilage matrix..$^{13}$ Detailed knowledge of cartilage thickness is also important for finite element work on synovial joints.

This paper presents the results of a study in which the cartilage thickness was surveyed over the entire surfaces of cadaveric lower limb joints. It further examines whether cartilage thickness had a correlation with its stiffness, and with any of the details of specimen donors such as age, weight, height, and body mass index. 


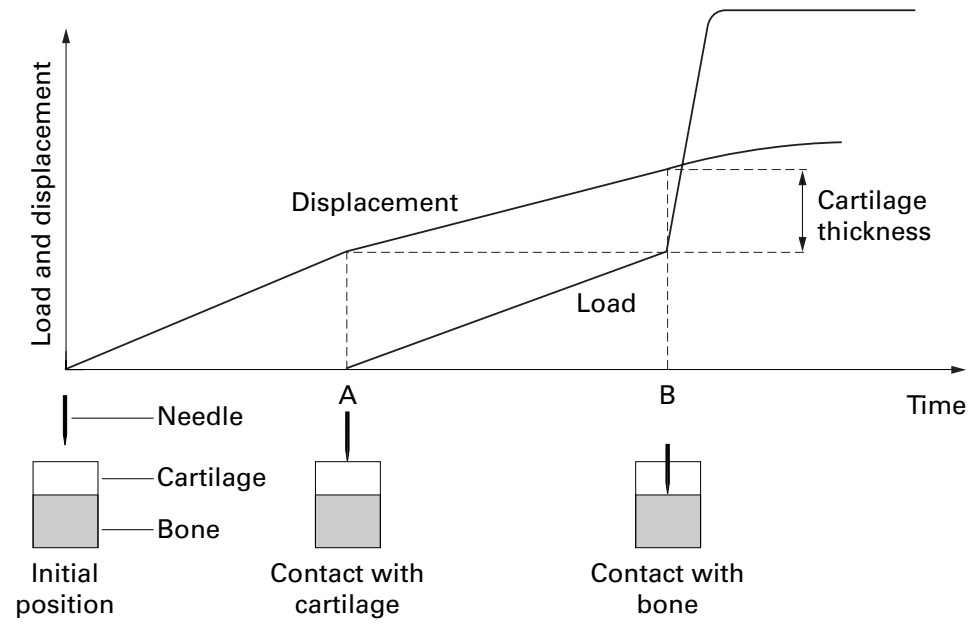

Figure 1 Response of load and displacement transducers for cartilage thickness measurement.

\section{Methods}

MATERIALS

Eleven sets of cadaveric human joints each comprising an ankle, knee, and hip were used in this study to measure the cartilage thickness over the whole surface of cadaveric lower limb joints. The joints within each set were obtained from the ipsilateral side and this meant that
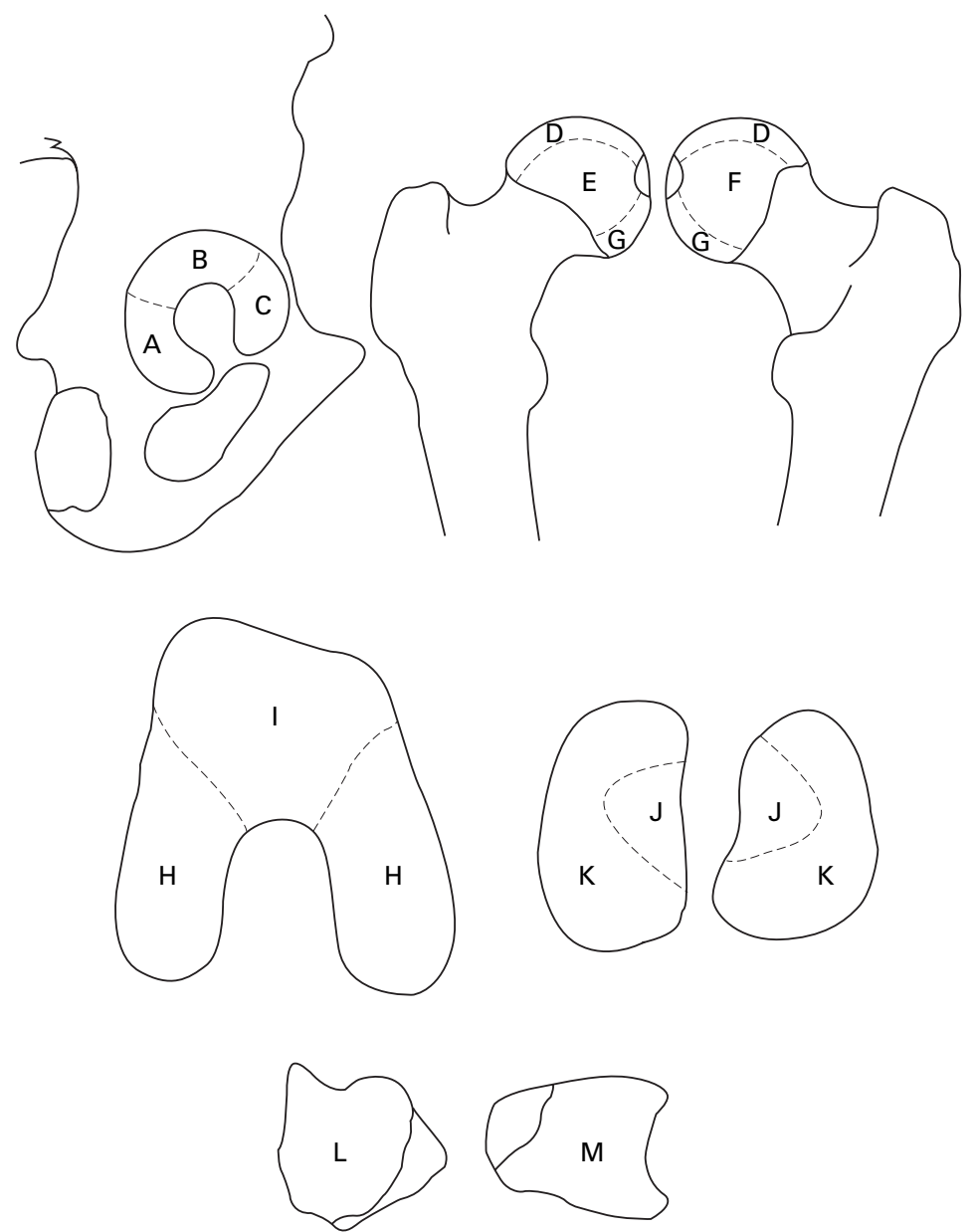

Figure 2 Distinct areas of the lower limb joints $(A, B, C$ : posterior, superior, and anterior areas of the acetabulum respectively; $D, E, F, G$ : superior, anterior, and posterior areas of the femoral head respectively; $H, I:$ patellar surface and femoral condyles respectively; $\mathcal{F}, K$ : tibial areas of the knee covered by the menisci and those that come into direct contact with the femur respectively; $L, M$ : talar and tibial areas of the ankle respectively).

each joint in a set would have been subjected to the same number of loading cycles. Table 1 gives details of the specimen donors. Availability was a major limiting factor on the number of this type of specimens.

\section{SPECIMEN PREPARATION}

Each set of joints was obtained from the mortuary within two to three days of death. The joints were sealed in plastic bags and stored at $-20^{\circ} \mathrm{C}$ in a freezer until the time of testing, when the joints were thawed overnight in a refrigerator at $4^{\circ} \mathrm{C}$. Once thawed, the surrounding tissue was removed from a joint so that the articular cartilage surfaces were exposed. A fine felt tip pen dipped in haematoxyline acid was used to draw a mesh over the entire articular surface of a joint yielding approximately 50 square test sites on the hip, 75 on the knee, and 35 on the ankle.

CARTILAGE THICKNESS MEASUREMENT

The cartilage thickness of each square (that did not show any visible signs of degeneration) on the mesh of all the articular surfaces was then measured using the technique of Swann and Seedhom. ${ }^{5}$

The method entails using an apparatus that allows a sharp needle to pierce the cartilage surface and move through the cartilage before coming to rest on the underlying bone at its interface with the cartilage. The apparatus also allowed simultaneous measurement to be taken of the displacement of the needle and of the load arising, from the moment the needle came into contact with the cartilage. From these measurements the cartilage thickness could be determined. The apparatus and technique have been previously described ${ }^{5}$ and so, only a brief account of the procedure is given here.

The specimen is placed approximately 0.5 $\mathrm{mm}$ below the needle. The needle assembly shaft is released and moves towards the cartilage before piercing the surface. Figure 1 shows a typical response of the load and displacement signals with time as the needle is allowed to move through the cartilage and into the underlying bone. Before releasing the needle, both the load and displacement signals have zero values. Once the needle is released it moves towards the cartilage surface and the displacement signal thus begins to increase, but the load signal remains at zero because the needle is not yet in contact with the cartilage surface. At the moment the needle comes into contact with the cartilage surface the load signal increases above zero, thus identifying the exact position of the displacement signal that corresponds to the cartilage surface (point A in fig 1).

The contact point with the underlying bone can also be determined. As the underlying bone is much stiffer than articular cartilage, there is a very rapid positive increase in the load signal when the needle reaches the bone interface with cartilage (point B). The cartilage thickness is the difference in displacement between the contact point with the bone (point B) and the contact point with the cartilage surface (point A). 
Table 2 Means and standard deviations of articular cartilage thickness of each ankle, knee, and hip within all 11 sets of joints and statistical comparisons. $p$ Values from t test

\begin{tabular}{|c|c|c|c|c|c|c|}
\hline \multirow[b]{2}{*}{ Set } & \multicolumn{3}{|c|}{ Mean (SD) mm } & \multicolumn{3}{|c|}{ Statistical comparisons } \\
\hline & Ankle & Knee & Hip & ank-kne & ank-hip & kne-hip \\
\hline 1 & $1.31(0.38)$ & $2.51(0.49)$ & $2.00(0.41)$ & $\mathrm{p}<0.001$ & $\mathrm{p}<0.001$ & $\mathrm{p}<0.001$ \\
\hline 2 & $1.14(0.17)$ & $1.99(0.48)$ & $1.65(0.41)$ & $\mathrm{p}<0.001$ & $\mathrm{p}<0.001$ & $\mathrm{p}<0.001$ \\
\hline 3 & $1.20(0.27)$ & $1.97(0.42)$ & $1.53(0.27)$ & $\mathrm{p}<0.001$ & $\mathrm{p}<0.001$ & $\mathrm{p}<0.001$ \\
\hline 4 & $1.62(0.25)$ & $2.51(0.40)$ & $1.71(0.60)$ & $\mathrm{p}<0.001$ & NS & $\mathrm{p}<0.001$ \\
\hline 5 & $1.25(0.22)$ & $1.99(0.37)$ & $1.54(0.27)$ & $\mathrm{p}<0.001$ & $\mathrm{p}<0.001$ & $\mathrm{p}<0.001$ \\
\hline 6 & $1.30(0.34)$ & $2.23(0.50)$ & $1.82(0.52)$ & $\mathrm{p}<0.001$ & $\mathrm{p}<0.001$ & $\mathrm{p}<0.001$ \\
\hline 7 & $1.19(0.34)$ & $1.80(0.42)$ & $1.40(0.23)$ & $\mathrm{p}<0.001$ & $\mathrm{p}<0.001$ & $\mathrm{p}<0.001$ \\
\hline 8 & $1.15(0.31)$ & $2.55(0.45)$ & $1.51(0.34)$ & $\mathrm{p}<0.001$ & $\mathrm{p}<0.001$ & $\mathrm{p}<0.001$ \\
\hline 9 & $1.30(0.29)$ & $2.17(0.53)$ & $1.67(0.33)$ & $\mathrm{p}<0.001$ & $\mathrm{p}<0.001$ & $\mathrm{p}<0.001$ \\
\hline 10 & $1.25(0.22)$ & $2.42(0.48)$ & $1.73(0.45)$ & $\mathrm{p}<0.001$ & $\mathrm{p}<0.001$ & $\mathrm{p}<0.001$ \\
\hline 11 & $1.00(0.20)$ & $1.69(0.39)$ & $1.35(0.30)$ & $\mathrm{p}<0.001$ & $\mathrm{p}<0.001$ & $\mathrm{p}<0.001$ \\
\hline
\end{tabular}

The load and displacement transducer signals were both sampled at $10 \mathrm{kHz}$ thus enabling accurate measurements of the cartilage thickness to be made. The needle had a velocity of $60 \mathrm{~mm} / \mathrm{sec}$ at the moment of contact with the cartilage surface and remains constant through the cartilage because there is very little resistance. As a sampling frequency of $10 \mathrm{kHz}$ was used, this would lead to an error of 0.006 $\mathrm{mm}$ in the measurement of cartilage thickness and as this was typically in the range 1 to $3 \mathrm{~mm}$, errors in the range 0.2 to $0.6 \%$ would be expected.

The repeatability of thickness measurements was previously investigated, using a similar apparatus, by Swann and Seedhom ${ }^{5}$ who measured the thickness of a rubber strip glued on to a metal plate at 17 sites. A mean thickness of $1.4 \mathrm{~mm}$ was found with a standard deviation of $0.017 \mathrm{~mm}$ giving a low coefficient of variation of $1.2 \%$. The mean value was also within $3 \%$ of the actual thickness of the rubber when measured directly with a micrometer.

STATISTICAL ANALYSIS OF THE CARTILAGE THICKNESS DATA

The means and standard deviations of the cartilage thickness were calculated for the whole surface of each ankle, knee, and hip within all 11 sets of joints. The cartilage thickness of each of these joints was found to be normally distributed as assessed using the AndersonDarling normality test. The one sided independent two sample $t$ test was therefore used to assess the significance of differences between the mean cartilage thickness of each ankle, knee, and hip within a set of joints.

The means and standard deviations of cartilage thickness were then determined for various distinct areas on each of the lower limb joints. The distinct areas on the hip were: the superior, anterior, posterior, and inferior areas of the femoral head, and the superior, anterior, and posterior areas of the acetabulum. The distinct areas of the knee were: the patellar and condylar surfaces of the femur, and on the tibia, those covered by the menisci and those areas that come into direct contact with the femur. The distinct areas of the ankle were those of the distal tibia and talus. Figure 2 shows these various distinct areas of the lower limb joints. The one sided independent two sample $t$ test was used to assess the significance of differences between the mean cartilage thickness of the distinct areas on each lower limb joint.

Simple linear regression analyses were carried out to see if any correlation existed between the mean cartilage thickness of each lower limb joint and the details of the specimen donors (age, mass, height, and body mass index).

The same type of analyses were also performed to examine if any correlation existed between cartilage thickness of each lower limb joint and the compressive modulus of the articular cartilage. The compressive modulus data were determined by Shepherd ${ }^{14}$ using an indentation technique described by Shepherd and Seedhom ${ }^{15}$ in which the load is applied to the cartilage under physiological loading conditions.

The statistical significance level was set at the $5 \%$ level $(\mathrm{p}<0.05)$ for all statistical tests.

\section{Results}

MEAN THICKNESS OF THE ENTIRE SURFACES OF THE LOWER LIMB JOINTS

Within all 11 sets of joints the ankle always had the thinnest cartilage whereas the knee always had the thickest. Table 2 shows the means and standard deviations of cartilage thickness of each ankle, knee, and hip within all 11 sets of joints. The ankles and knees had mean cartilage thickness in the ranges 1.0 to 1.62 $\mathrm{mm}$ and 1.69 to $2.55 \mathrm{~mm}$ respectively, while the hips had mean cartilage thickness in the range 1.35 to $2.0 \mathrm{~mm}$.

Table 2 also shows statistical comparisons of the mean cartilage thickness of each ankle, knee, and hip within a set of joints. The mean cartilage thickness of the knee was significantly greater than that of both the ankle and hip $(p<0.001)$ within each sets of the 11 joints, while the cartilage thickness of the hip was significantly greater than that of the ankle within 10 of these sets $(p<0.001)$.

\section{TOPOGRAPHICAL VARIATION IN CARTILAGE} THICKNESS

\section{Hip}

(a) Distinct areas of the femoral head

Four distinct areas on each femoral head, the superior, anterior, posterior, and inferior areas were compared. They had mean cartilage thickness in the ranges 1.41 to $2.25 \mathrm{~mm}, 1.46$ to $2.09 \mathrm{~mm}, 1.26$ to $1.98 \mathrm{~mm}$, and 1.08 to 2.4 $\mathrm{mm}$ respectively. The means and standard 
Table 3 Means and standard deviations of articular cartilage thickness of the distinct areas of each femoral head and acetabulum and statistical comparisons. $p$ Values from $t$ test

\begin{tabular}{|c|c|c|c|c|c|c|c|c|c|c|}
\hline \multirow[b]{3}{*}{ Set } & \multicolumn{10}{|l|}{ Femoral head } \\
\hline & \multicolumn{4}{|c|}{ Mean (SD) $m m$} & \multicolumn{6}{|c|}{ Statistical comparisons } \\
\hline & Superior & Anterior & Posterior & Inferior & Sup-Ant & Sup-Pos & Sup-Inf & Ant-Pos & Ant-Inf & Pos-Inf \\
\hline 1 & $1.61(0.13)$ & $2.09(0.44)$ & - & $2.40(0.00)$ & $\mathrm{p}<0.05$ & - & - & - & - & - \\
\hline 2 & $1.78(0.49)$ & $1.67(0.34)$ & $1.70(0.17)$ & $1.08(0.12)$ & NS & NS & $\mathrm{p}<0.05$ & NS & $\mathrm{p}<0.01$ & $\mathrm{p}<0.01$ \\
\hline 3 & $1.63(0.38)$ & $1.83(0.00)$ & $1.40(0.18)$ & $1.52(0.08)$ & - & NS & NS & - & - & NS \\
\hline 4 & $2.25(0.79)$ & $1.75(0.57)$ & $1.98(0.54)$ & $1.51(0.09)$ & NS & NS & $\mathrm{p}<0.05$ & NS & NS & $\mathrm{p}<0.05$ \\
\hline 5 & $1.78(0.25)$ & $1.58(0.28)$ & $1.56(0.20)$ & $1.50(0.21)$ & NS & NS & $\mathrm{p}<0.05$ & NS & NS & NS \\
\hline 6 & $2.22(0.50)$ & $2.03(0.42)$ & $1.73(0.45)$ & $1.32(0.24)$ & NS & $\mathrm{p}<0.05$ & $\mathrm{p}<0.001$ & NS & $\mathrm{p}<0.01$ & $\mathrm{p}<0.05$ \\
\hline 7 & $1.53(0.15)$ & $1.62(0.27)$ & $1.29(0.12)$ & $1.38(0.25)$ & NS & $\mathrm{p}<0.001$ & NS & $\mathrm{p}<0.01$ & NS & NS \\
\hline 8 & $1.43(0.42)$ & - & $1.48(0.15)$ & $1.70(0.01)$ & - & NS & NS & - & - & $\mathrm{p}<0.05$ \\
\hline 9 & $1.67(0.27)$ & $1.78(0.29)$ & $1.60(0.32)$ & $1.69(0.48)$ & NS & NS & NS & NS & NS & NS \\
\hline 10 & $2.09(0.49)$ & $1.73(0.41)$ & $1.65(0.37)$ & $1.32(0.03)$ & $\mathrm{p}<0.05$ & $\mathrm{p}<0.01$ & $\mathrm{p}<0.001$ & NS & $\mathrm{p}<0.001$ & $\mathrm{p}<0.01$ \\
\hline 11 & $1.41(0.29)$ & $1.46(0.30)$ & $1.26(0.15)$ & $1.31(0.15)$ & NS & $\mathrm{p}<0.05$ & NS & $\mathrm{p}<0.01$ & $\mathrm{p}<0.05$ & NS \\
\hline
\end{tabular}

No comparison was possible because of severe degeneration of the cartilage where no statistical comparison is shown.

deviations of the cartilage thickness of these distinct areas are shown in table 3 together with the statistical comparisons of the mean cartilage thickness of the distinct areas on each femoral head.

Significant differences in mean cartilage thickness between the superior and anterior areas on the femoral head only occurred in sets 1 and 10 . With set 1 , the anterior area was significantly thicker than the posterior $(p<0.05)$, while set number 10 showed the superior area to be significantly thicker than that of the anterior $(\mathrm{p}<0.05)$.

In four of the hip joints the superior area had cartilage that was significantly thicker than that of the posterior area $(\mathrm{p}<0.001$ to $\mathrm{p}<0.05)$, while in five hip joints the superior area was significantly thicker than the inferior area $(\mathrm{p}<0.001$ to $\mathrm{p}<0.05)$. The anterior area was found to have significantly thicker cartilage than the posterior area in two hip joints $(p<0.01)$ and the inferior area in four hip joints $(\mathrm{p}<0.001$ to $\mathrm{p}<0.05)$.

Comparison of the cartilage thickness of the posterior and inferior areas showed that the posterior area had significantly thicker cartilage in four hip joints $(\mathrm{p}<0.01$ to $\mathrm{p}<0.05)$, while the inferior area had significantly thicker cartilage in one of the hip joints $(\mathrm{p}<0.05)$.

(b) Distinct areas of the acetabulum

Three distinct areas of each acetabulum were compared. These were the superior, anterior, and posterior areas and they had mean cartilage thickness in the ranges 1.24 to 2.25 $\mathrm{mm}, 1.2$ to $1.71 \mathrm{~mm}$, and 1.24 to $1.85 \mathrm{~mm}$ respectively. Table 3 shows the means and standard deviations of the cartilage thickness of these distinct areas.

Referring again to Table 3, it can be seen that only in two hip joints were any significant differences found in the mean cartilage thickness between the superior, anterior, and posterior areas of the acetabulum. In sets 2 and 9 the superior area had significantly thicker cartilage than the anterior area $(p<0.01$ to $p<0.05)$. The posterior area was found to be significantly thicker than the anterior area $(\mathrm{p}<0.01)$ in set 2 .

\section{Knee}

(a) Distinct areas of the femur

Two distinct areas of the femur were compared. These were the patellar surface and the femoral condyles and they had mean cartilage thickness in the ranges of 1.76 to $2.59 \mathrm{~mm}$ and 1.65 to $2.65 \mathrm{~mm}$ respectively. The means and standard deviations of the cartilage thickness of the distinct areas of each femur are shown in table 4 together with the statistical comparisons.

Five knee joints showed no significant difference in thickness between the patellar surface and the femoral condyles. In four knee joints the patellar surface had significantly thicker cartilage than the femoral condyles $(p<0.001$ to $\mathrm{p}<0.05$ ) and in one knee joint (set 8 ) the femoral condyles had significantly thicker cartilage than the patellar surface $(p<0.001)$. No comparison of cartilage thickness was possible for set number 6 because the patellar surface had full thickness cartilage loss and no areas could be tested.

Table 4 Means and standard deviations of articular cartilage thickness of the distinct areas of each femur and tibia and statistical comparisons. $p$ Values from $t$ test

\begin{tabular}{|c|c|c|c|c|c|c|}
\hline \multirow[b]{3}{*}{ Set } & \multicolumn{3}{|l|}{ Femur } & \multicolumn{3}{|l|}{ Tibia } \\
\hline & \multicolumn{2}{|l|}{ Mean (SD) $m m$} & \multirow{2}{*}{$\frac{\text { Stat }}{\text { Con-Pat }}$} & \multicolumn{2}{|c|}{ Mean (SD) $m m$} & \multirow{2}{*}{$\frac{\text { Stat }}{\text { Cov-Dir }}$} \\
\hline & Patellar surface & Condyles & & Covered & Direct & \\
\hline 1 & $2.59(0.34)$ & $2.61(0.48)$ & NS & $2.15(0.46)$ & $2.87(0.49)$ & $\mathrm{p}<0.01$ \\
\hline 2 & $2.36(0.42)$ & $2.05(0.47)$ & $\mathrm{p}<0.05$ & $1.66(0.39)$ & $2.13(0.26)$ & $\mathrm{p}<0.05$ \\
\hline 3 & $1.99(0.29)$ & $1.83(0.30)$ & NS & $1.97(0.35)$ & $2.69(0.70)$ & $\mathrm{p}<0.05$ \\
\hline 4 & $2.49(0.32)$ & $2.49(0.33)$ & NS & $2.37(0.49)$ & $2.83(0.46)$ & $\mathrm{p}<0.05$ \\
\hline 5 & $2.28(0.28)$ & $2.00(0.38)$ & $\mathrm{p}<0.01$ & $1.79(0.30)$ & $2.07(0.37)$ & $\mathrm{p}<0.05$ \\
\hline 6 & - & $2.20(0.45)$ & - & $2.27(0.61)$ & - & - \\
\hline 7 & $1.95(0.30)$ & $1.65(0.29)$ & $\mathrm{p}<0.001$ & $1.65(0.29)$ & $2.63(0.60)$ & $\mathrm{p}<0.01$ \\
\hline 8 & $2.06(0.35)$ & $2.65(0.34)$ & $\mathrm{p}<0.001$ & $2.53(0.44)$ & $2.98(0.26)$ & $\mathrm{p}<0.01$ \\
\hline 9 & $2.47(0.29)$ & $1.99(0.45)$ & $\mathrm{p}<0.001$ & $2.06(0.61)$ & $2.56(0.61)$ & $\mathrm{p}<0.05$ \\
\hline 10 & $2.32(0.19)$ & $2.53(0.45)$ & NS & $2.09(0.37)$ & $2.94(0.22)$ & $\mathrm{p}<0.001$ \\
\hline 11 & $1.76(0.34)$ & $1.63(0.31)$ & NS & $1.54(0.32)$ & $2.20(0.51)$ & $\mathrm{p}<0.001$ \\
\hline
\end{tabular}

No comparison was possible because of severe degeneration of the cartilage where no statistical comparison is shown. 


\begin{tabular}{|c|c|c|c|c|c|}
\hline \multicolumn{6}{|l|}{ Acetabulum } \\
\hline \multicolumn{3}{|c|}{ Mean (SD) $m m$} & \multicolumn{3}{|c|}{ Statistical comparisons } \\
\hline Superior & Anterior & Posterior & Sup-Ant & Sup-Pos & Ant-Pos \\
\hline $2.25(0.24)$ & $1.71(0.45)$ & - & NS & - & - \\
\hline $1.95(0.42)$ & $1.34(0.08)$ & $1.85(0.29)$ & $\mathrm{p}<0.01$ & NS & $\mathrm{p}<0.01$ \\
\hline $1.42(0.02)$ & $1.45(0.20)$ & - & NS & - & - \\
\hline $1.35(0.34)$ & $1.33(0.09)$ & $1.31(0.22)$ & NS & NS & NS \\
\hline $1.52(0.28)$ & $1.31(0.16)$ & $1.28(0.28)$ & NS & NS & NS \\
\hline $1.51(0.64)$ & $1.62(0.23)$ & $1.55(0.41)$ & NS & NS & NS \\
\hline $1.29(0.21)$ & $1.37(0.29)$ & $1.24(0.17)$ & NS & NS & NS \\
\hline $1.49(0.28)$ & $1.46(0.74)$ & $1.71(0.45)$ & NS & NS & NS \\
\hline $1.80(0.39)$ & $1.48(0.21)$ & $1.61(0.16)$ & $\mathrm{p}<0.05$ & NS & NS \\
\hline $1.64(0.37)$ & $1.49(0.37)$ & $1.50(0.20)$ & NS & NS & NS \\
\hline $1.24(0.43)$ & $1.20(0.15)$ & $1.36(0.26)$ & NS & NS & NS \\
\hline
\end{tabular}

(b) Distinct areas of the tibia

Two distinct areas of the tibia were compared. These were the areas covered by the menisci and those that come into direct contact with the femur. The former had mean cartilage thickness in the ranges 1.54 to $2.53 \mathrm{~mm}$ and the latter 2.07 to $2.98 \mathrm{~mm}$. The means and standard deviations of the cartilage thickness of these distinct areas of each tibia are shown in table 4 together with the statistical comparisons of the mean cartilage thickness of the same areas.

Within 10 knee joints, cartilage on those areas which come into direct contact with the femur was significantly thicker than cartilage on those areas covered by the menisci $(\mathrm{p}<0.001$ to $\mathrm{p}<0.05)$. A comparison of the areas of the tibia covered by the menisci with those that come into direct contact with the
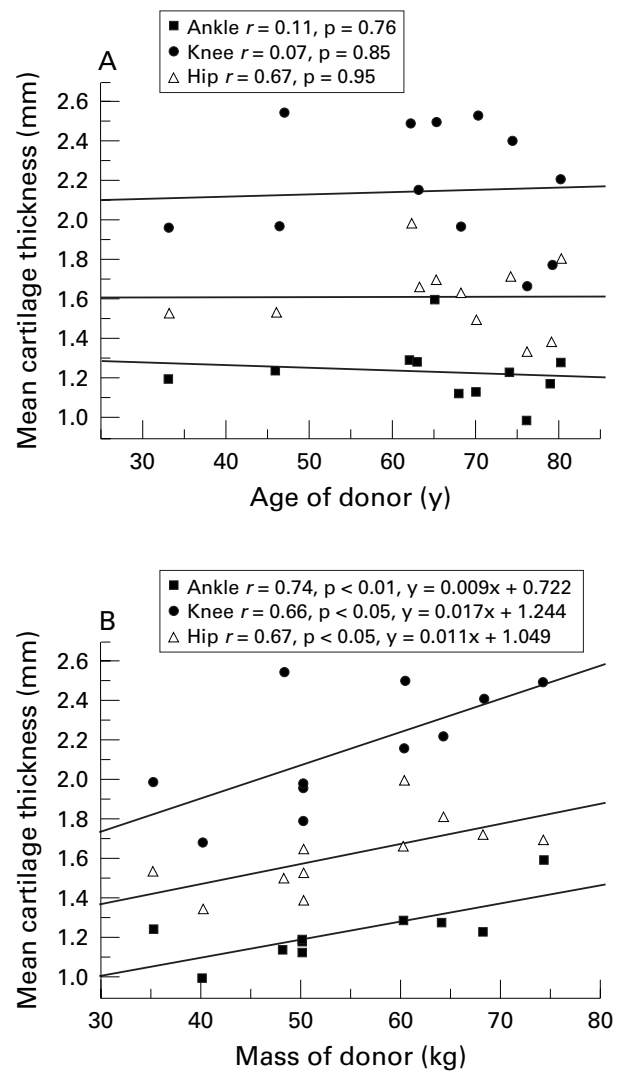

femur was not possible for the knee in set number 6 because the cartilage on the latter areas was degenerate and no measurements were possible.

Ankle

In the 11 ankle joints the tibial and talar surfaces had mean cartilage thickness in the ranges of 1.06 to $1.63 \mathrm{~mm}$ and 0.94 to 1.62 $\mathrm{mm}$ respectively. Table 5 shows the means and standard deviations of the cartilage thickness of the tibial and talar surfaces within each ankle joint. The statistical comparisons of the mean cartilage within each ankle joint are also shown in table 5 .

In seven ankle joints the tibia had significantly thicker cartilage than the talus $(p<0.001$ to $\mathrm{p}<0.05$ ), while the remaining four ankle joints showed no significant difference in mean cartilage thickness between the tibial and the talar surfaces.

CORRELATIONS OF CARTILAGE THICKNESS WITH DETAILS OF SPECIMEN DONORS

Simple linear regression analyses were carried out to examine if any correlations existed between the mean cartilage thickness of various joints and the details of the specimen donors (age, mass, height, and body mass index).

No correlation was found between the age of donors and the mean cartilage thickness of any of the joints (fig 3A). However, significant correlations were found between the mass of donors and the mean cartilage thickness for each joint as can be seen in figure $3 \mathrm{~B}$. The coefficients of correlation and levels of signifi-
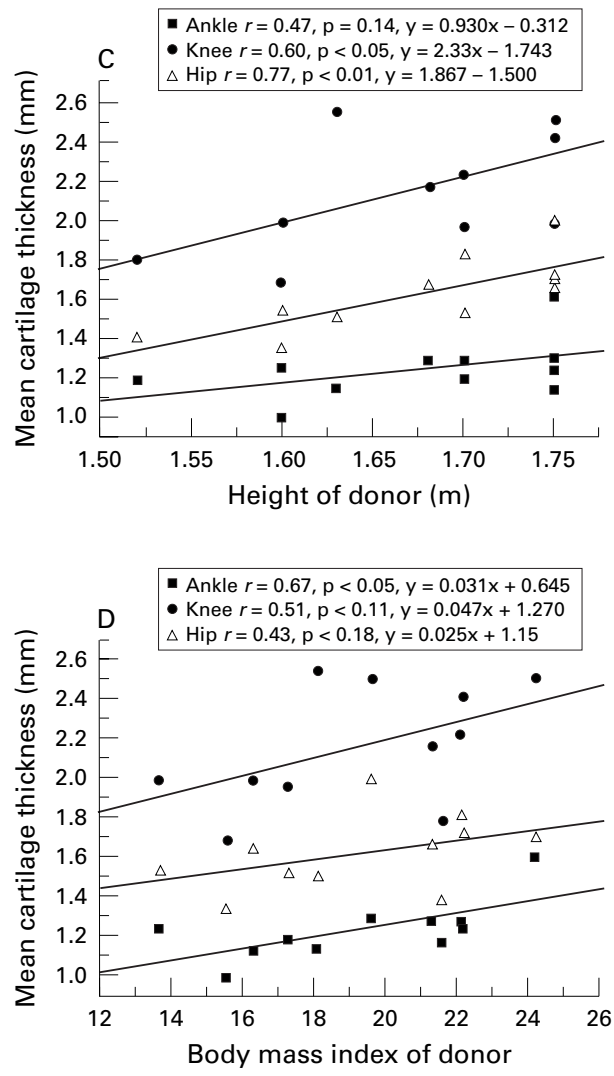

Figure 3 Variation of mean cartilage thickness of the lower limb joints with details of the specimen donors. (A) age, (B) mass, (C) height, (D) body mass index. 

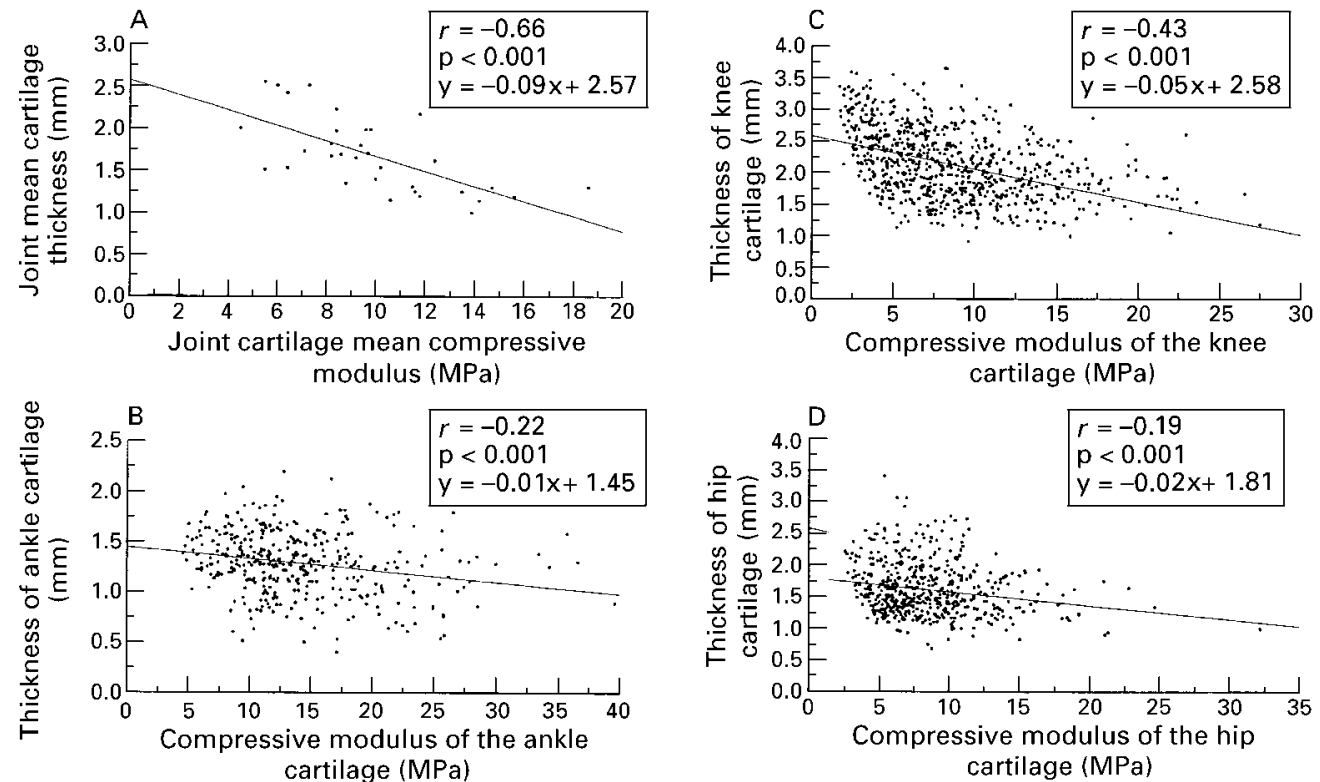

Figure 4 Variation of cartilage thickness modulus. (A) Each ankle, knee, and hip, (B) each site on all ankles, (C) each site on all knees, (D) each site on all hips.

cance between the mass of donors and the mean cartilage thickness of the ankle, knee, and hip joints were $r=0.74$ at $\mathrm{p}<0.01, r=0.66$ at $\mathrm{p}<0.05$, and $r=0.67$ at $\mathrm{p}<0.05$ respectively.

A significant correlation was also found between the height of donors and the mean cartilage thickness of the knee and hip joints as shown in figure 3C. The coefficients of correlation and levels of significance between the height of specimens and the mean cartilage thickness of the knee and hip joints were $r=0.6$ at $\mathrm{p}<0.05$ and $r=0.77$ at $\mathrm{p}<0.01$ respectively.

Furthermore, a significant correlation was found between the body mass index of the donors and the mean cartilage thickness of the ankle joint, as can be seen in figure 3D. The coefficient of correlation and level of significance between the body mass index of donors and the mean cartilage thickness of the ankle joint was $r=0.67$ at $\mathrm{p}<0.05$.

CARTILAGE THICKNESS AND CARTILAGE COMPRESSIVE MODULUS

The mean cartilage thickness of each ankle, knee, and hip was plotted against its mean articular cartilage compressive modulus (fig $4 \mathrm{~A})$. The data suggest an inverse relation between the thickness of articular cartilage and

Table 5 Means and standard deviations of articular cartilage thickness of the distinct areas of each ankle and statistical comparisons. $p$ Values from t test

\begin{tabular}{|c|c|c|c|}
\hline \multirow[b]{2}{*}{ Set } & \multicolumn{2}{|c|}{ Mean $(S D) m m$} & \multirow{2}{*}{$\frac{\text { Stat }}{\text { Tib-Tal }}$} \\
\hline & Tibia & Talus & \\
\hline 1 & $1.57(0.23)$ & $1.16(0.37)$ & $\mathrm{p}<0.01$ \\
\hline 2 & $1.18(0.12)$ & $1.11(0.19)$ & NS \\
\hline 3 & $1.33(0.14)$ & $1.09(0.30)$ & $\mathrm{p}<0.01$ \\
\hline 4 & $1.63(0.15)$ & $1.62(0.29)$ & NS \\
\hline 5 & $1.23(0.13)$ & $1.28(0.30)$ & NS \\
\hline 6 & $1.45(0.17)$ & $1.12(0.39)$ & $\mathrm{p}<0.001$ \\
\hline 7 & $1.34(0.11)$ & $1.11(0.39)$ & $\mathrm{p}<0.01$ \\
\hline 8 & $1.31(0.24)$ & $1.05(0.31)$ & $\mathrm{p}<0.01$ \\
\hline 9 & $1.44(0.18)$ & $1.07(0.27)$ & $\mathrm{p}<0.001$ \\
\hline 10 & $1.26(0.13)$ & $1.24(0.26)$ & NS \\
\hline 11 & $1.06(0.17)$ & $0.94(0.21)$ & $\mathrm{p}<0.05$ \\
\hline
\end{tabular}

its compressive modulus. That is, thin cartilage has a high compressive modulus and thick cartilage a low compressive modulus.

A more detailed examination of the data was made using the cartilage thickness and compressive modulus data of each individual indentation test site from a joint surface, as shown in figures $4 \mathrm{~B}, 4 \mathrm{C}$, and $4 \mathrm{D}$. Again the data confirm an inverse relation between the cartilage thickness and its compressive modulus. While the low $\mathrm{p}$ values indicate significance, the relatively low $r$ values indicate a high scatter in the data.

\section{Discussion}

CARTILAGE THICKNESS AND JOINT CONGRUENCE WITH REFERENCE TO THE LOWER LIMB JOINTS Examining the cartilage thickness data showed that the knee had significantly thicker cartilage than both the ankle and hip in all 11 sets of joints, while in 10 sets of joints the hip had significantly thicker cartilage than the ankle. Few explanations have been given in the medical literature as to the differences in cartilage thickness in joints, but Simon ${ }^{2}$ proposed that the thickness of articular cartilage is related to the congruence of the joint; thin cartilage is found in congruent joints, whereas thick cartilage is found in incongruent joints.

In another study Simon et $a l^{16}$ measured the congruence ratio (average length of the congruent surface divided by the average length of the total articular surface) and thickness of articular cartilage in the joints of dogs. They found that the knee had the thickest cartilage, the hip less thick, and the ankle thinner cartilage still as was also found in this study for human lower limb joints. An inverse relation was found between cartilage thickness and congruence ratio of the joint with thick cartilage correlating with low congruence of the joint and thin cartilage with high congruence.

Simon et $a l^{16}$ suggested that the correlation between cartilage thickness and congruence 
ratio of the joint tends to equalise the stress in congruent and incongruent joints. Congruent joints with thin cartilage only deform a small amount, yet the area of contact is large enough to distribute the load and maintain an acceptable level of stress. With incongruent joints deformation of the thick cartilage increases the contact area between the joint surfaces sufficiently to decrease the stress to an acceptable level.

CORRELATIONS OF CARTILAGE THICKNESS WITH DETAILS OF SPECIMEN DONORS

In this study the mean cartilage thickness of the lower limb joints were found to correlate with some of the details of the specimen donors. The mass of donors was found to correlate with the mean cartilage thickness of all three lower limb joints. Correlations were also found between the height of donors and the mean cartilage thickness of the knee and hip joints, while only in the ankle joint was a correlation found between the mean cartilage thickness and the body mass index of donors. These correlations imply that the larger and heavier a donor was the thicker the cartilage in the lower limb joints.

A possible explanation of this is given by Simon $^{13}$ who determined the thickness of articular cartilage in animals ranging in size from the mouse to the cow. He found that the thickness of articular cartilage generally increased with body size and there was a linear logarithmic relation between cartilage thickness and body weight. The same author concluded that the thickness of articular cartilage was scaled from small to large animals, according to the law of simple allometry. The law related growth of part of an organism to that of the whole. This could explain the positive correlations found in this study between the cartilage thickness of some of the lower limb joints and the mass, height and body mass index of the specimen donors. Hall and Wyshak ${ }^{17}$ also found a correlation between the thickness of articular cartilage in the knee joint and the weight of patients, yet Armstrong and Gardner $^{18}$ could find no correlation between the thickness of femoral head cartilage and the body weight of specimen donors.

Although correlations were found between the cartilage thickness and some details of the specimen donors it should be highlighted that the samples in this study were mainly from elderly donors. The mean (SD) age of the donors was 65 (14) years and this should be kept in mind when considering the results. Indeed, this may explain why Armstrong and Gardner ${ }^{18}$ found no correlation between the thickness of femoral head cartilage and the body weight of specimen donors because their specimens had an extended age range from 10 to 68 years.

CORRELATIONS OF CARTILAGE THICKNESS WITH ITS COMPRESSIVE MODULUS

In this study the data suggest an inverse relation between cartilage thickness and compressive modulus when the mean values of each ankle, knee, and hip are examined. When a more detailed examination was made of the site specific data of the cartilage thickness and compressive modulus, the low $\mathrm{p}$ values indicated significance yet the $r$ values indicated a high scatter in the data.

Few studies have examined the correlation between the thickness of articular cartilage and its compressive modulus, but some published data suggest that no correlation exists. Simon ${ }^{13}$ showed that cartilage thickness does not have a direct correlation with static compressive stress on the joint surface. Athanasiou et al ${ }^{19}{ }^{20}$ found no correlation between aggregate modulus of articular cartilage and its thickness. These two studies considered knee joint cartilage from five species (bovine, canine, human, monkey, and rabbit) and human hip cartilage respectively. Another more recent study ${ }^{21}$ on the cartilage thickness in 15 pairs of ipsilateral human knees and ankles showed that cartilage thickness was significantly correlated with its stiffness expressed as the $2 \mathrm{~s}$ creep modulus. The present study, which is in agreement with this latter study, has also the advantage that sets of joints (ankle, knee, and hip) were obtained from the ipsilateral side and therefore the thickness of cartilage from knee joints from one population was not compared with hip or ankle joints from a different population.

The authors would like to thank Messrs M Pullan and B Whitham for their technical expertise and help with the experiments. This work was facilitated by a PhD scholarship from the University of Leeds.

1 Kempson GE, Freeman MAR, Swanson SAV. The determination of a creep modulus for articular cartilage from indentation tests on the human femoral head. J Biomech 1971;4:239-50.

2 Simon WH. Scale effects in animal joints. II: Thickness and elasticity in the deformability of articular cartilage. Arthrielasticity in the deformability of

3 Parsons JR, Black J. The viscoelastic shear behaviour of normal rabbit articular cartilage. J Biomech 1977;10:21-9.

4 Jurvelin J, Arokoski J, Kirivanta I, Tammi M, Helminen HJ. Indentation study of the biomechanical properties of articular cartilage in the canine knee. Engng Med 1987;16: $15-22$.

5 Swann AC, Seedhom BB. Improved techniques for measuring the indentation and thickness of articular cartilage. Proc Inst Mech Eng [H] 1989;203:143-50.

6 Rushfeldt PD, Mann RW, Harris WH. Improved techniques for measuring in vitro the geometry and pressure distribution in the human acetabulum. 1. Ultrasonic measurement of the acetabular surfaces, sphericity and cartilage thickness. J Biomech 1981;14:253-61.

7 Modest VE, Murphy MC, Mann RW. Optical verification of a technique for in situ ultrasonic measurement of articular cartilage thickness. J Biomech 1989;22:171-6.

8 Myers SL, Dines K, Brandt DA, Brandt KD, Albrecht ME. Experimental assessmeny by high frequency ultrasound of
articular cartilage thickness and osteoarthritic changes. J Rheumatol 1995;22:109-16.

9 Jurvelin JS, Rasanen T, Kolmonen P, Lyyra T. Comparison of optical, needle probe and ultrasonic techniques for the measurement of articular cartilage thickness. J Biomech 1995;28:231-5

10 Yao JQ, Seedhom BB. Ultrasonic measurements of articular cartilage. Connect Tissue Res 1997;36:143.

11 Kladny B, Bail H, Swododa B, Schiwy-Bochat H, Beyer WF, Weseloh G. Cartilage thickness measurement in magnetic resonance imaging. Osteoarthritis Cartilage 1996;4:181-6.

12 Eckstein F, Adam C, Sittek H, Becker C, Milz S, Schulte E, et al. Non-invasive determination of cartilage thickness throughout joint surfaces using magnetic resonance imaging. J Biomech 1997;30:285-9.

13 Simon WH. Scale effects in animal joints. I Articular cartilage thickness and compressive stress. Arthritis Rheum $1970 ; 13: 244-55$. 
14 Shepherd DET. The compressive modulus of human articular cartilage in joints of the lower limb determined under physiologi-
cal loading conditions. [ $\mathrm{PhD}$ thesis]. Leeds: University of cal loading cond

15 Shepherd DET, Seedhom BB. A technique for measuring the compressive modulus of articular cartilage under physiological loading rates with preliminary results. Proc Inst Mech Eng [H] 1997;211:155-65.

16 Simon WH, Friedenberg S, Richardson S. A correlation of joint congruence and thickness of articular cartilage in dogs. J Bone Joint Surg [Am] 1973;55:1614-20.

17 Hall FM, Wyshak G. Thickness of articular cartilage in the normal knee. J Bone Joint Surg [Am] 1980;62:408-13.
18 Armstrong CG, Gardner DL. Thickness distribution of human femoral head articular cartilage. Ann Rheum Dis human femoral hea

19 Athanasiou KA, Rosenwasser MP, Buckwalter JA, Malinin TI, Mow VC. Interspecies comparisons of in situ intrinsic mechanical properties of distal femoral cartilage. J Orthop Res 1991;9:330-40.

20 Athanasiou KA, Agarwal A, Dzida FJ. Comparative study of the intrinsic mechanical properties of the human acetabular and femoral head cartilage. J Orthop Res 1994;12:340-9.

21 Yao JQ, Seedhom BB. Topographical variations in the cartilage of human ankle and knee joints. J Bone Joint Surg $[\mathrm{Br}]$ 1997;79:457.

\section{Historical images}

Series editors: W Grassi, C Cervini
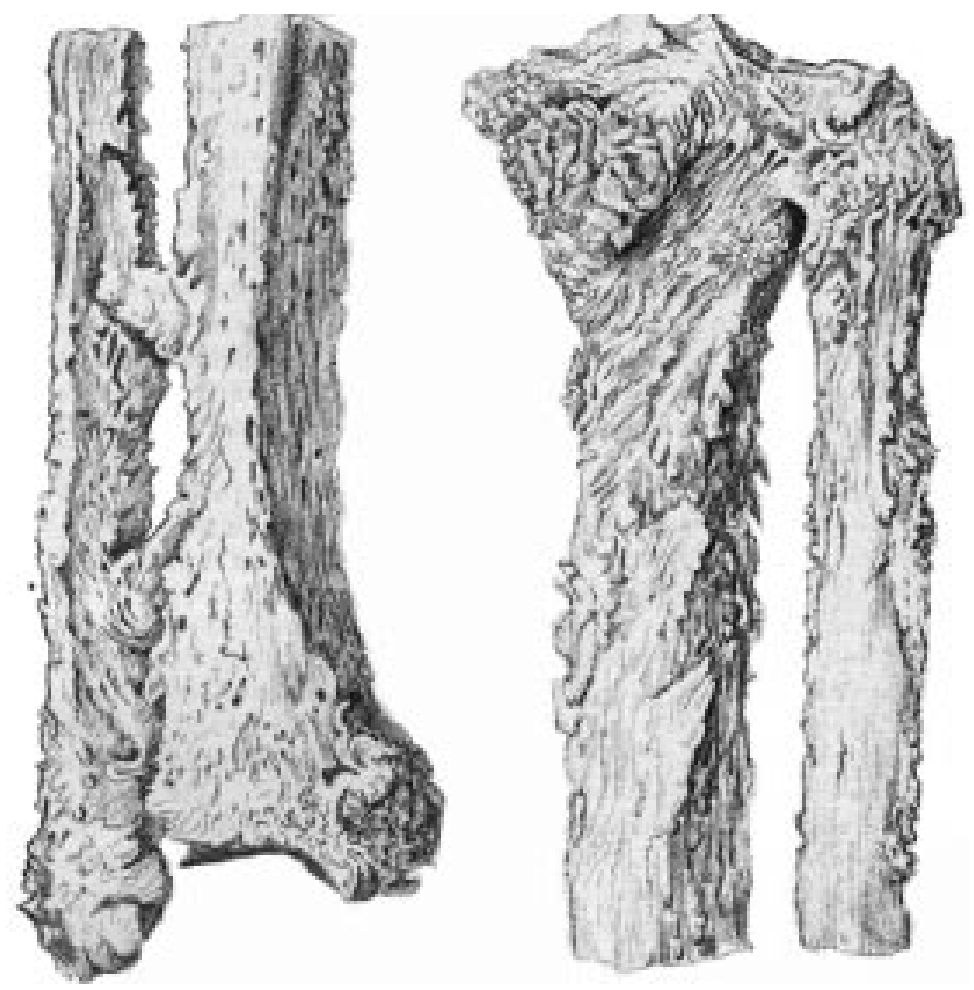

Figure 12 Hypertrophic osteopathy. Bernard L.

Dystrophies osseuses acquises d'origine obscure. In: Marfan A-B, Apert, Aviragnet, Bernard L, Garnier M, Hallé F, Milian. Maladies des os. Paris: Librairie f-B Baillière et fils, 1912. 